\title{
Changes in liver stiffness and its associated factors during oral antiviral therapy in Chinese patients with chronic hepatitis B
}

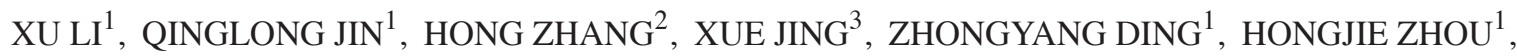 \\ ZETIAN ZHANG ${ }^{1}$, DONGQING YAN ${ }^{1}$, DONGMEI LI ${ }^{1}$, PUJUN GAO ${ }^{1}$ and JUNQI NIU ${ }^{1}$ \\ ${ }^{1}$ Department of Hepatology; ${ }^{2}$ Phase I Clinical Trials Unit, The First Hospital of Jilin University, \\ Changchun, Jilin 130021; ${ }^{3}$ Department of Gastroenterology, The Affiliated Hospital of Medical College, \\ Qingdao University, Qingdao, Shandong 266000, P.R. China
}

Received September 15, 2015; Accepted October 28, 2016

DOI: $10.3892 /$ etm.2017.4065

\begin{abstract}
The present study aimed to assess improvements in liver stiffness determined by transient elastography and associated factors in Chinese patients with chronic hepatitis B (CHB) during long-term treatment with oral antiviral drugs. A total of 334 consecutive Chinese patients with $\mathrm{CHB}$ who underwent oral antiviral therapy and received at least two liver stiffness measurements (LSMs) at the First Hospital of Jilin University (Changchun, China) from December 2012 to February 2015 were enrolled in the present study. The cohort included 201 patients without liver cirrhosis (group 0) and 133 patients with liver cirrhosis (group 1). Each patient was subjected to LSM twice with an interval of 6 months. The mean initial liver stiffness values were 14.01 \pm 9.37 and $21.59 \pm 10.25 \mathrm{kPa}$ for patients in group 0 and group 1 , respectively $(\mathrm{P}<0.001)$. Multivariate analysis revealed that higher aspartate aminotransferase and lower alanine aminotransferase levels at baseline as well as higher $\alpha$-fetoprotein levels at follow-up ( 24 weeks) were associated with a greater decline of liver stiffness in group 0. Furthermore, a higher liver stiffness at baseline and a longer course of antiviral therapy prior to the initial LSM were significantly correlated with a reduction of liver stiffness, whereas higher total bilirubin levels at follow-up contributed to increased liver stiffness in group 1. In conclusion, LSM at the beginning and the end of a 24-week observation period showed that antiviral drug therapy significantly improved in group 1 , while a marked decreasing trend was also observed in group 0 . In group 0 , the reduction of liver stiffness was correlated with liver inflammation, whereas in group 1, it was correlated with the treatment duration prior to the initial LSM and serum levels of hepatitis B virus DNA.
\end{abstract}

Correspondence to: Professor Pujun Gao or Professor Junqi Niu, Department of Hepatology, The First Hospital of Jilin University, 71 Xinmin Street, Changchun, Jilin 130021, P.R. China

E-mail: gpj0411@163.com

E-mail: 15143166188@139.com

Key words: liver fibrosis, anti-viral drug, chronic hepatitis B
Furthermore, a higher liver stiffness at baseline was associated with a greater reduction of liver stiffness in each group.

\section{Introduction}

Approximately 2 billion individuals have chronic hepatitis $\mathrm{B}$ virus (HBV) infection, of whom $\sim 350$ million have chronic hepatitis B (CHB) (1). Its complications conclude cirrhosis, hepatic decompensation and hepatocellular carcinoma. In $23 \%$ of patients with $\mathrm{CHB}$ progression to liver cirrhosis occurs due to long-term hepatic necroinflammation. The ultimate goal regarding the treatment of CHB is to block the advancement of chronic liver injury to the stages of cirrhosis, hepatic decompensation and/or hepatocellular carcinoma (HCC). However, it is now commonly known that liver cirrhosis can be reversed by treatment with nucleoside/nucleotide analogs (NAs), particularly in the early stage $(2,3)$.

Liver fibrosis measurement using transient elastography (TE) has gained increasing attention globally. TE utilizes an ultrasound (US) transducer attached to the axis of the vibrator that generates pulsation featuring a mild amplitude and low frequency $(50 \mathrm{~Hz})$. The resultant elastic shear wave passes through the liver, and detection based on US pulse-echo tracks the dissemination of the shear wave and computes its velocity, which is positively proportional to liver tissue stiffness (4-6).

Five oral drugs are licensed for the treatment of $\mathrm{CHB}$ infection: Lamivudine (LAM), entecavir (ETV), telbivudine (LdT), adefovir dipivoxil (ADV) and tenofovir disoproxil fumarate (TDF). The present study evaluated the effects of long-term NA treatment on liver injury and hepatic fibrosis in 334 Chinese CHB patients who underwent liver stiffness measurement (LSM) twice using FibroScan at baseline and at week 24 of the treatment. LSM values in liver cirrhosis patients significantly improved and a clear decreasing trend was observed in non-cirrhotic patients after 24 weeks of oral anti-viral therapy.

\section{Materials and methods}

Patients. A total of 334 consecutive Chinese CHB patients (who were hepatitis B surface antigen-positive for $\geq 6$ months) were enrolled in the present study from December 2012 to 
February 2015. These patients included 226 (67.7\%) patients who used ETV, 32 patients $(9.6 \%)$ patients who used LAM, 31 patients $(9.3 \%)$ who used ADV, 18 patients (5.4\%) who used LdT and 27 patients (8.1\%) who used TDF. These patients received at least two LSMs at the First Hospital of Jilin University (Changchun, China), while being administered oral antiviral drugs.

Patients who were positive for antibodies to human immunodeficiency virus or for HCV and patients who had excessive alcohol consumption or drug abuse were excluded. In addition, patients with a Child Pugh Score of $\geq 9$ or HCC indicated by the first abdominal US examination were excluded.

Upon enrollment, each patient was subjected to an initial LSM, and a second LSM was performed $\sim 6$ months later. Liver cirrhosis was identified by ultrasonography.

The recruitment of human participants and the protocol of the present study were approved by the Independent Institutional Review Board of the First Hospital of Jilin University (Changchun, China). An informed consent form was signed by each participant prior to enrollment.

Laboratory tests. All serological, biochemical and virological examinations were completed within 5 days prior to LSM. Blood samples collected upon admission were subjected to the ARCHITECT HBV surface antigen (HBsAg) assay (Abbott Laboratories, Chicago, IL, USA); serum hepatitis B e antigen (HBeAg) levels were determined using a microparticle enzyme immunoassay (ARCHITECT i2000; Abbott Laboratories). Furthermore, serum HBV DNA levels were established using the COBAS TaqMan HBV test with the High Pure System (Roche Molecular Diagnostics, Inc., Pleasanton, CA, USA) at a lower detection limit of $15 \mathrm{IU} / \mathrm{ml}$. Serum HBV DNA levels were expressed as $\log 10$ units. Alanine aminotransferase (ALT), aspartate aminotransferase (AST) and total bilirubin (TBIL) were measured by dry chemistry methods (Johnson \& Johnson, New Brunswick, NJ, USA). $\alpha$-fetoprotein (AFP) was determined by chemiluminescence (Roche Molecular Diagnostics, Inc.). Prothrombin time (PT) was assessed by a coagulation method (Sysmex Corporation, Kobe, Japan) and prothrombin time activity (PTA) was calculated using the following formula: PTA $(\%)=\left[\mathrm{PT}_{\text {control }}-\left(\mathrm{PT}_{\text {control }} \mathrm{x} 0.6\right)\right] /\left[\mathrm{PT}_{\text {test }}-\left(\mathrm{PT}_{\text {control }} \mathrm{x} 0.6\right)\right] \times 100$.

LSM. LSM was performed using TE with an M-probe (FibroScan; Echosens, Paris, France). Prior to LSM, patients had fasted overnight. A minimum of 10 valid measurements were acquired from each patient. LSM values were only considered valid and entered into the final analysis if the success rate of LSM measurements was $>60 \%$. Calculated median values of the valid measurements (expressed in $\mathrm{kPa}$ ), reflected the relative liver stiffness for each patient at a specific time-point.

Statistical analysis. Quantitative differences in liver stiffness between two points were analyzed by Student's $t$-test. Percentages were weighed by the Chi-square test. Continuous variables were expressed as the mean \pm standard deviation. Reductions of liver stiffness were compared using simple linear regression and multivariate analyses were performed using multiple linear regression, with adjustment for the covariates gender, age, AST, ALT, TBIL, AFP, HBV DNA, initial liver stiffness and the course of antiviral therapy prior to the initial LSM. All tests were two-sided and $\mathrm{P}<0.05$ was considered to indicate a statistically significant difference. SPSS version 18.0 (SPSS, Inc., Chicago, IL, USA) and SAS V.9.1 (SAS Institute, Inc., Cary, NC, USA) were used for all statistical analyses.

\section{Results}

Patient characteristics at baseline. Clinical and laboratory characteristics of participants at baseline and follow-up are shown in Table I. A total of $334 \mathrm{CHB}$ patients treated with NAs were enrolled in the present study, including 250 males and 84 females with a mean age of 47.49 years. Patients were assigned to group 0 when liver cirrhosis was absent $(n=201)$ and to group 1 when liver cirrhosis was present $(n=133)$.

No significant difference between the two groups was detected in demographic characteristics, including gender and family history of HBV infection. Furthermore, serum HBV DNA titer as well as HBeAg-negative rate were not different between the two groups. However, the mean age in group 1 was higher than that in group $0(49.41 \pm 9.36$ vs. $46.22 \pm 9.60$ years, $\mathrm{P}=0.003)$. Furthermore, group 1 featured higher levels of baseline and follow-up ALT (43.04 \pm 45.19 vs. $39.25 \pm 56.89 \mathrm{IU} / 1, \mathrm{P}=0.002 ; 36.12 \pm 26.32$ vs. $26.11 \pm 14.09$ IU/1, $\mathrm{P}<0.001)$, AST $(53.47 \pm 47.06$ vs. $42.09 \pm 42.78 \mathrm{IU} / 1$, $\mathrm{P}<0.001 ; 34.32 \pm 20.01$ vs. $23.87 \pm 8.49 \mathrm{IU} / 1, \mathrm{P}<0.001)$, TBIL (31.41 \pm 46.65 vs. $22.59 \pm 14.86 \mu \mathrm{mol} / 1, \mathrm{P}=0.003 ; 26.17 \pm 14.83$ vs. $19.93 \pm 9.82 \mu \mathrm{mol} / 1, \mathrm{P}<0.001)$, AFP levels $(34.51 \pm 143.49$ vs. $20.65 \pm 72.11 \mathrm{ng} / \mathrm{ml}, \mathrm{P}=0.005 ; 18.19 \pm 111.93$ vs. $3.13 \pm 1.77 \mathrm{ng} / \mathrm{ml}$, $\mathrm{P}<0.001)$ and liver stiffness $(21.59 \pm 10.25$ vs. $14.01 \pm 9.37 \mathrm{kPa}$, $\mathrm{P}<0.001 ; 15.76 \pm 9.52$ vs. $10.29 \pm 28.64 \mathrm{kPa}, \mathrm{P}<0.001)$ compared to those in group 0 . In addition, the course of drug treatment prior to the initial LSM in group 1 was shorter than that in group 0 (566.47 \pm 785.38 vs. $832.34 \pm 896.33$ days; $\mathrm{P}=0.002)$. However, group 0 had higher initial qHBsAg levels $(2850.23 \pm 4914.02$ vs. $1208.87 \pm 1303.47 \mathrm{IU} / \mathrm{ml} ; \mathrm{P}=0.035)$ and PTA than group 1 $(88.49 \pm 12.72$ vs. $75.23 \pm 14.98 \% ; \mathrm{P}<0.001)$.

Changes in liver biochemical markers and stiffness value. Biochemical markers significantly improved after patients in group 0 underwent long-term oral antiviral therapy. For instance, initial and follow-up levels were 42.09 \pm 42.78 and $23.87 \pm 8.49 \mathrm{IU} / 1$ for $\mathrm{AST}(\mathrm{P}<0.001), 39.25 \pm 56.89$ and $26.11 \pm 14.09 \mathrm{IU} / 1$ for ALT $(\mathrm{P}=0.002), 22.59 \pm 14.86$ and $19.93 \pm 9.82 \mu \mathrm{mol} / 1$ for TBIL $(\mathrm{P}=0.012)$ and $20.65 \pm 72.11$ and $3.13 \pm 1.77 \mathrm{ng} / \mathrm{ml}$ for AFP $(\mathrm{P}=0.001)$. The mean liver stiffness value at baseline was $14.01 \pm 9.37 \mathrm{kPa}$, which decreased to $10.29 \pm 28.64 \mathrm{kPa}$ at follow-up; however, the difference was not significant $(\mathrm{P}=0.077)$.

Initial and follow-up levels in group 1 were 53.47 \pm 47.06 and $34.32 \pm 20.01 \mathrm{IU} / 1$ for $\mathrm{AST}(\mathrm{P}<0.001), 43.04 \pm 45.19$ and $36.12 \pm 26.32 \mathrm{IU} / 1$ for ALT $(\mathrm{P}=0.116), 31.41 \pm 46.65$ and $26.17 \pm 14.83 \mu \mathrm{mol} / 1$ for TBIL $(\mathrm{P}=0.191)$ and $34.51 \pm 143.49$ and $18.19 \pm 111.93 \mathrm{ng} / \mathrm{ml}$ for AFP $(\mathrm{P}=0.179)$. The liver stiffness value significantly decreased from $21.59 \pm 10.25$ to $15.76 \pm 9.52 \mathrm{kPa}$ $(\mathrm{P}<0.001)$ during the 24 weeks of NA therapy.

Parameters correlated with the improvement of liver stiffness. In group 0, univariate analysis suggested that a higher liver stiffness at baseline is a factor indicating a greater 
Table I. Clinical and laboratory findings for the two groups.

\begin{tabular}{|c|c|c|c|}
\hline Characteristic & Group $0(n=201)$ & Group 1 (n=133) & P-value \\
\hline Age (years) & $46.22 \pm 9.60$ & $49.41 \pm 9.36$ & 0.003 \\
\hline Male gender, n (\%) & $152(76)$ & $98(74)$ & 0.690 \\
\hline \multicolumn{4}{|l|}{ AST (IU/1) } \\
\hline Baseline & $42.09 \pm 42.78$ & $53.47 \pm 47.06$ & $<0.001$ \\
\hline Follow-up & $23.87 \pm 8.49^{\mathrm{a}}$ & $34.32 \pm 20.01^{\mathrm{a}}$ & $<0.001$ \\
\hline \multicolumn{4}{|l|}{ ALT (IU/1) } \\
\hline Baseline & $39.25 \pm 56.89$ & $43.04 \pm 45.19$ & 0.002 \\
\hline Follow-up & $26.11 \pm 14.09^{\mathrm{b}}$ & $36.12 \pm 26.32$ & $<0.001$ \\
\hline \multicolumn{4}{|l|}{ TBIL $(\mu \mathrm{mol} / \mathrm{l})$} \\
\hline Baseline & $22.59 \pm 14.86$ & $31.41 \pm 46.65$ & 0.003 \\
\hline Follow-up & $19.93 \pm 9.82^{\mathrm{b}}$ & $26.17 \pm 14.83$ & $<0.001$ \\
\hline \multicolumn{4}{|l|}{ PTA $(\%)$} \\
\hline Baseline & $88.49 \pm 12.72$ & $75.23 \pm 14.98$ & $<0.001$ \\
\hline Follow-up & $92.1 \pm 13.09$ & $79.24 \pm 15.16$ & $<0.001$ \\
\hline \multicolumn{4}{|l|}{$\operatorname{AFP}(n g / m l)$} \\
\hline Baseline & $20.65 \pm 72.11$ & $34.51 \pm 143.49$ & 0.005 \\
\hline Follow-up & $3.13 \pm 1.77^{\mathrm{b}}$ & $18.19 \pm 111.93$ & $<0.001$ \\
\hline \multicolumn{4}{|l|}{ Liver stiffness value $(\mathrm{kPa})$} \\
\hline Baseline & $14.01 \pm 9.37$ & $21.59 \pm 10.25$ & $<0.001$ \\
\hline Follow-up & $10.29 \pm 28.64$ & $15.76 \pm 9.52^{\mathrm{a}}$ & $<0.001$ \\
\hline \multicolumn{4}{|l|}{ HBV infection history (n) } \\
\hline Yes/no & $91 / 110$ & $65 / 68$ & 0.519 \\
\hline \multicolumn{4}{|l|}{$\operatorname{HBeAg}(\mathrm{n})$} \\
\hline Positive/negative & $96 / 105$ & $52 / 81$ & 0.119 \\
\hline Baseline HBV DNA $\left(\log _{10} \mathrm{IU} / \mathrm{ml}\right)$ & $2.45 \pm 1.73$ & $2.65 \pm 1.66$ & 0.121 \\
\hline Baseline qHBsAg (IU/ml) & $2,850.23 \pm 4,914.02$ & $1,208.87 \pm 1,303.47$ & 0.035 \\
\hline Therapy prior to initial LSM (days) & $832.34 \pm 896.33$ & $566.47 \pm 785.38$ & 0.002 \\
\hline
\end{tabular}

decline of liver stiffness $(\mathrm{P}<0.001)$, while multivariate analysis suggested that higher AST levels $(>40 \mathrm{IU} / \mathrm{l} ; \mathrm{P}<0.001)$ and lower ALT levels $(\leq 40 \mathrm{IU} / 1 ; \mathrm{P}<0.001)$ at baseline as well as higher AFP levels $(>15 \mathrm{ng} / \mathrm{ml})$ at follow-up $(\mathrm{P}=0.031)$ are correlated with a significant reduction in liver stiffness (Table II).

In group 1, univariate analysis revealed that parameters that may predict a significant reduction of the liver stiffness value included a higher serum HBV DNA titer (>2000 IU/ml; $\mathrm{P}=0.001)$, higher serum AFP levels $(>15 \mathrm{ng} / \mathrm{ml})$ at baseline $(\mathrm{P}=0.045)$, a higher initial liver stiffness value $(\mathrm{P}<0.001)$ and a longer course of antiviral therapy prior to the initial LSM $(\mathrm{P}=0.040)$. Multivariate analysis revealed that lower total bilirubin levels $(\leq 17.2 \mu \mathrm{mol} / \mathrm{l})$ at follow-up $(\mathrm{P}=0.005)$, a higher liver stiffness value at baseline $(\mathrm{P}<0.001)$ and a longer course of antiviral therapy prior to the initial LSM $(\mathrm{P}=0.003)$ were correlated with a significant reduction of liver stiffness values (Table III).

\section{Discussion}

CHB remains a global healthcare challenge, is complicated by the development of cirrhosis and hepatocarcinoma and accounts for $\sim 600,000$ mortalities per year (7).

Maximization of viral suppression with the objective of controlling liver fibrosis and preventing progression to clinical complications is the aim of treating chronic HBV infection (8). Certain studies have demonstrated that the degree of liver fibrosis in patients with $\mathrm{CHB}$ can be decreased by treatment with antiviral drugs. For instance, liver fibrosis improved in $35-38 \%$ of lamivudine-treated CHB patients and 36-39\% of ETV-treated CHB patients after 1 year $(9,10)$. Long-term ETV treatment ( $>3$ years) attenuated necroinflammatory activity in all patients and reduced liver fibrosis in 57-100\% of patients $(11,12)$.

Liver biopsy provides liver tissue for assessing liver pathology, but its applications are limited due to its invasiveness, 


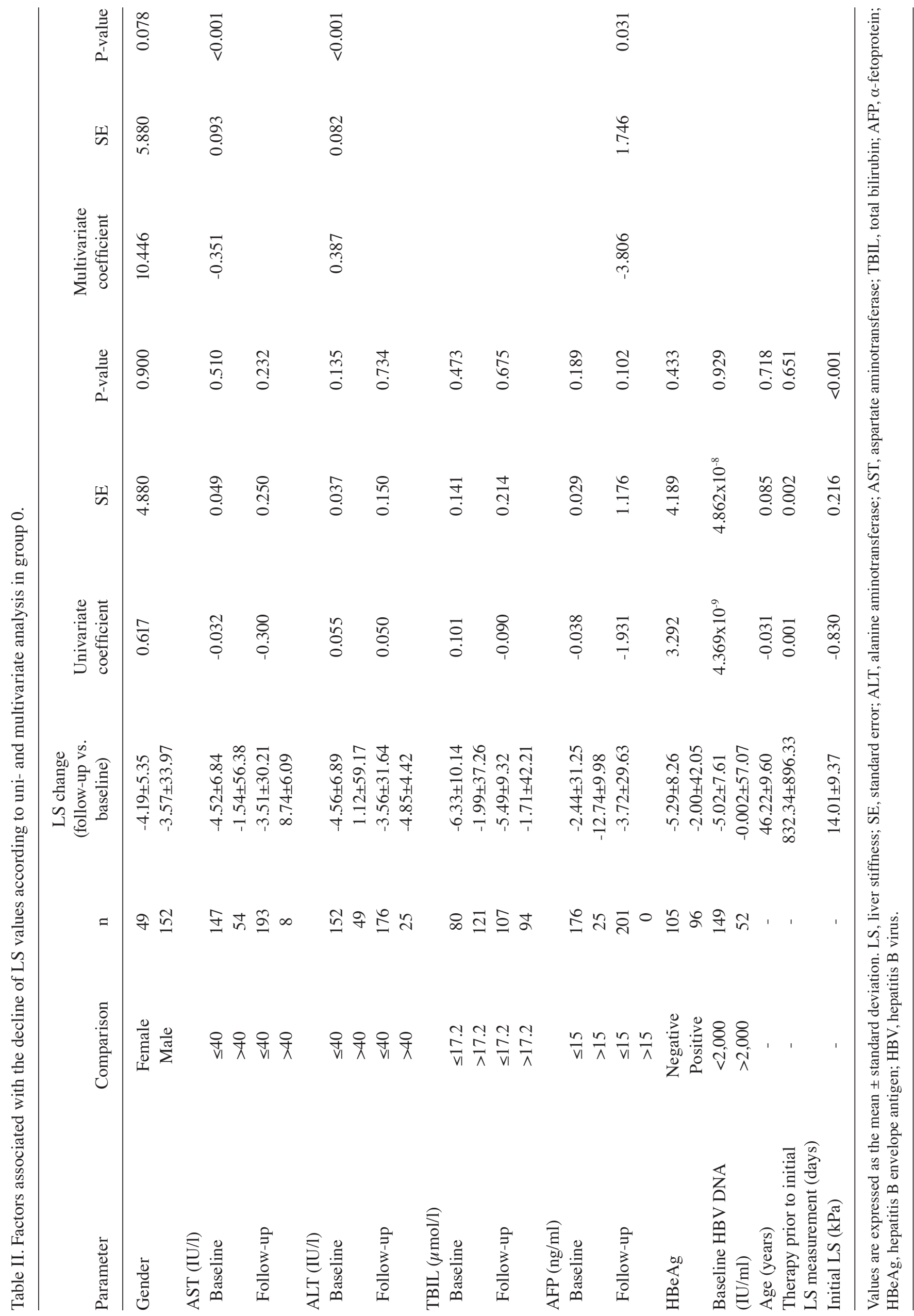




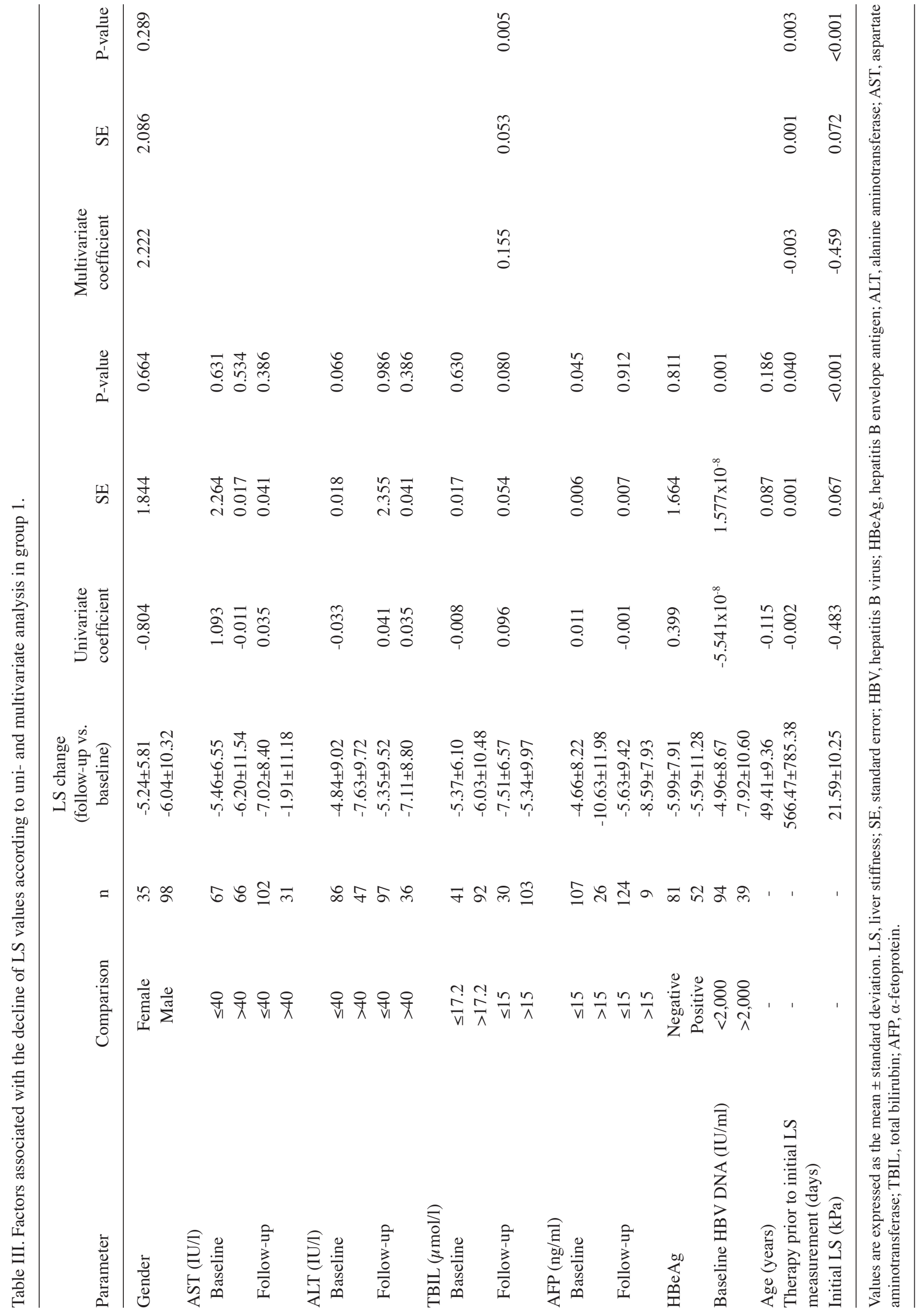


patient discomfort, sampling errors, inter-observer variation and risk for complications of internal bleeding $(13,14)$. LSM is one of the increasingly popular non-invasive methods for detecting and grading liver fibrosis. Numerous studies have revealed that this method has good reproducibility, relative reflection of histology and clinical utility (14).

The present study investigated the impact of 24 weeks of oral antiviral therapy on liver stiffness in 334 patients. Each patient was evaluated by TE at baseline as well as at the end of the 24-week period. The results revealed that liver injury and inflammation had significantly improved. The results further confirmed the efficacy of oral antiviral treatment in mitigating liver histopathology along with potent inhibition of HBV DNA replication.

In group 0, a significant reduction of AST, ALT, TBIL and AFP levels was observed after antiviral therapy. However, the liver stiffness value did not show a significant decline. This may be due to the fact that patients without liver cirrhosis had a better liver condition than those in group 1 when they were enrolled and their hepatic histological changes are based on necroinflammation. Wong et al (15) prospectively studied patients with $\mathrm{CHB}$ undergoing liver biopsy twice before and at week 48 of antiviral treatment, revealing a significant decrease of the metavir fibrosis score, while the metavir activity score showed no significant change.

In group 1, a significant decline of the liver stiffness value and AST levels was observed. However, there were no significant changes in the levels of ALT, TBIL and AFP, which is different from the results of a previous study (16). This may be due to the interval time between two examinations being 6 months in the present study, which is shorter than that used previously. It appears that the necroinflammation requires more time to improve than fibrosis when liver cirrhosis is present.

In addition, the present study demonstrated that the decline of LSM values was associated with several virological and biochemical factors in CHB patients, which were principally consistent with previously published data (16).

In group 0, univariate analysis suggested that a higher liver stiffness value at baseline indicates a greater decrease of the liver stiffness value. Multivariate analysis showed that patients who had higher AFP levels at follow-up as well as higher AST and lower ALT levels at baseline achieved a greater decline in liver stiffness. This indicated that, in non-cirrhotic $\mathrm{CHB}$ patients, the improvement of liver stiffness is associated with liver inflammation at baseline and follow-up. Furthermore, factors associated with poor liver parenchyma appeared to contribute to a greater decline of liver stiffness. This correlation was in agreement with the findings by Kuo et al (16) on the effects of ETV on liver stiffness.

Similarly, in group 1, a higher liver stiffness at baseline contributed to a greater reversal of fibrosis, which is in accordance with the results on patients without liver cirrhosis. In addition, higher serum AFP levels at baseline and a higher initial HBV DNA titer were correlated with a significant reduction of liver stiffness. Longer antiviral therapy duration prior to the initial LSM may predict a significant reduction of liver stiffness. Furthermore, multivariate analysis revealed that longer antiviral therapy duration prior to the initial LSM was associated with an improvement in liver fibrosis. It appears that in cirrhotic $\mathrm{CHB}$ patients, the duration of oral NA intake and the amount of viral replication significantly affect the efficacy of antiviral therapy, which is supported by the findings by Osakabe et al (11). The main limitation of the present study was that no liver histology was available for this cohort. Secondly, leading to a small number of subjects in our study, the impact of AST levels were not considered when patients were included, there is a need for further studies in large patients population which considering the impact of aminotransferase.

In conclusion, the present study evaluated changes in liver stiffness in $334 \mathrm{CHB}$ patients who received oral antiviral treatment in a 24-week interval and found a significant decrease in liver stiffness values in patients with cirrhosis, while a decreasing trend was also observed in non-cirrhotic patients. Higher liver stiffness values at baseline contributed to a greater reduction of liver stiffness in each group. In patients without cirrhosis, the reduction of liver stiffness was associated with liver inflammation, whereas it was associated with treatment duration prior to the first LSM and serum HBV DNA levels in patients with liver cirrhosis.

\section{Acknowledgements}

The authors would like to thank Dr Hongqin Xu (Department of Hepatology, The First Hospital of Jilin University) for her assistance in statistical evaluation for the study.

\section{References}

1. Gish RG: Clinical trial results of new therapies for HBV: Implications for treatment guidelines. Semin Liver Dis 25 (Suppl 1): S29-S39, 2005.

2. Lee S and Kim DY: Non-invasive diagnosis of hepatitis B virus-related cirrhosis. World J Gastroenterol 20: 445-459, 2014.

3. Su TH, Kao JH and Liu CJ: Molecular mechanism and treatment of viral hepatitis-related liver fibrosis. Int J Mol Sci 15: 10578-10604, 2014.

4. Friedrich-Rust M, Ong MF, Martens S, Sarrazin C, Bojunga J, Zeuzem S and Herrmann E: Performance of transient elastography for the staging of liver fibrosis: A meta-analysis. Gastroenterology 134: 960-974, 2008.

5. Barreiro P, Labarga P, Martín-Carbonero L, Amor A, Ruiz-Sancho A, Castellares C, González-Lahoz J and Soriano V: Sustained virological response following HCV therapy is associated with non-progression of liver fibrosis in HCV/HIV-coinfected patients. Antivir Ther 11: 869-877, 2006.

6. Sandrin L, Fourquet B, Hasquenoph J, Yon S, Fournier C, Mal F, Christidis C, Ziol M, Poulet B and Kazemi F: Transient elastography: A new noninvasive method for assessment of hepatic fibrosis. Ultrasound Med Biol 29: 1705-1713, 2003.

7. Gill US and Kennedy PT: New insights in the management of chronic hepatitis B. Clin Med (Lond) 15: 191-196, 2015.

8. Marcellin P, Gane E, Buti M, Afdhal N, Sievert W, Jacobson IM, Washington MK, Germanidis G, Flaherty JF, Aguilar Schall R, et al: Regression of cirrhosis during treatment with tenofovir disoproxil fumarate for chronic hepatitis B: A 5-year open-label follow-up study. Lancet 381: 468-475, 2013.

9. Chang TT, Gish RG, de Man R, Gadano A, Sollano J, Chao YC, Lok AS, Han KH, Goodman Z, Zhu J, et al: A comparison of entecavir and lamivudine for HBeAgpositive chronic hepatitis B. N Engl J Med 354: 1001-1010, 2006.

10. Lai CL, Shouval D, Lok AS, Chang TT, Cheinquer H, Goodman Z, DeHertogh D, Wilber R, Zink RC, Cross A, et al: Entecavir versus lamivudine for patients with $\mathrm{HBeAgnegative}$ chronic hepatitis B. N Engl J Med 354: 1011-1020, 2006.

11. Osakabe K, Ichino N, Nishikawa T, Sugiyama H, Kato M, Kitahara S, Hashimoto S, Kawabe N, Harata M, Nitta Y, et al: Reduction of liver stiffness by antiviral therapy in chronic hepatitis B. J Gastroenterol 46: 1324-1334, 2011. 
12. Schiff ER, Lee SS, Chao YC, Kew Yoon S, Bessone F, Wu SS, Kryczka W, Lurie Y, Gadano A, Kitis G, et al: Long-term treatment with entecavir induces reversal of advanced fibrosis or cirrhosis in patients with chronic hepatitis B. Clin Gastroenterol Hepatol 9: 274-276, 2011.

13. Bedossa P, Dargére D and Paradis V: Sampling variability of liver fibrosis in chronic hepatitis C. Hepatology 38: 1449-1457, 2003

14. Ganne-Carrié N, Ziol M, de Ledinghen V, Douvin C, Marcellin P, Castera L, Dhumeaux D, Trinchet JC and Beaugrand M: Accuracy of liver stiffness measurement for the diagnosis of cirrhosis in patients with chronic liver diseases. Hepatology 44: 1511-1517, 2006.
15. Wong GL, Wong VW, Choi PC, Chan AW, Chim AM, Yiu KK, Chu SH, Chan FK, Sung JJ and Chan HL: On-treatment monitoring of liver fibrosis with transient elastography in chronic hepatitis B patients. Antivir Ther 16: 165-172, 2011.

16. Kuo YH, Lu SN, Chen CH, Chang KC, Hung CH, Tai WC, Tsai MC, Tseng PL, Hu TH and Wang JH: The changes of liver stiffness and its associated factors for chronic hepatitis B patients with entecavir therapy. PLoS One 9: e93160, 2014. 